\title{
Effects of LSD on auditory generalization*
}

\author{
LINDA A. DYKSTRA and JAMES B. APPEL \\ University of Chicago. Chicago, Ul. 60637
}

Rats were trained on a two-response discrete-trial pure-tone discrimination (500 and $1.000 \mathrm{~Hz}$ ). Generalization was tested by randomly varying the frequency of the auditory stimuli $(200-1,300 \mathrm{~Hz})$ and by observing the resulting changes in response probability. Doses of .04- and $.16 \mathrm{mg} \mathrm{LSD} / \mathrm{kg}$ produced consistent effects on reaction time but did not change the shape of the generalization gradient.

In man, hallucinogenic drugs such as d-lysergic acid diethylamide (LSD) have been reported to induce a "sharpened sense of hearing [Hollister, 1968]." If "sharpened" means an increased disposition to respond to auditory stimuli, a similar effect occurs in cats; LSD causes an increase in behavioral and EEG arousal produced by tones of different intensities (Key \& Bradley, 1960; Bradley \& Elkes, 1957; Bradley \& Key, 1959). There is, however, no evidence that increased arousal, attention, or even auditory acuity affects ability to discriminate on the basis of sound, i.e., respond differentially (appropriately) to some change in the physical properties of auditory stimuli. One way to measure this ability is to examine an animal's disposition to respond in the presence of other stimuli along the same dimension as the training stimuli, i.e., generalization (Terrace, 1966). The accuracy of the original discrimination can then be measured by the slope of the obtained generalization "gradient." A flat gradient, i.e., nondifferential responding to all test stimuli, indicates little discrimination, whereas a steep gradient, i.e., maximal responding at the training stimulus, indicates that the animal is under good stimulus control.

When an attempt was made to investigate the effects of LSD $\mathrm{cn}$ this measure in cats, it was found that the drug increased the number of trials necessary to extinguish a conditioned avoidance response in a shuttlebox but did not alter the shape of the generalization gradient (Key, 1961). In the present experiment, these effects were studied with a different species (rats), response (barpressing), and reinforcer (milk). A discrete-trial two-choice procedure was used to minimize the confounding effects of changes in the animal's rate of responding (Blough, 1963; Migler, 1964; Migler \& Millenson, 1969).

* This research was supported by Research Grant 5-R01-MH-13,186, Predoctoral Research Fellowship 1-F01-MH-48508-01 (L.A.D.) and Research Scientist Development Award 1-K2-MH-09355 (J.B.A.) from the National Institute of Mental Health and by Grant 102-13-RD from the Illinois Department of Nental Health.

\section{SUBJECTS}

The Ss were three male albino rats of the Sprague-Dawley strain obtained from the Charles River Breeding Laboratories, Wilmington, Massachusetts. At the beginning of the experiment. they were approximately 120 days old and weighed $300 \mathrm{~g}$. Their food intake was restricted such that their weights remained at a relatively constant level. About $10 \mathrm{~g}$ of Purina Laboratory Chow was given each day, in addition to whatever liquid food was obtained in the apparatus. Water was always available in individual home cages. The animals were housed in a room kept on a 12-h day-night cycle (7:00 a.m. to 7:00 p.m.) with constant temperature $\left(75^{\circ} \mathrm{F}\right)$ and humidity $(40 \%-50 \%)$.

\section{APPARATUS}

The apparatus consisted of a standard Lehigh Valley chamber (Model 1316), housed in a sound- and light-attenuated shell (LVE Model 1316C). An exhaust fan in the shell masked laboratory and electronic noises. The chamber was illuminated by a shaded $10-\mathrm{W}$ bulb and contained two 2 -in. levers, located 2 in. above the right and left sides of a $1 \frac{3 / 4}{4} \mathrm{cu}$-in. food magazine. The levers protruded $1 / 2$ in. from the front panel. A diet of $0.05 \mathrm{ml}$ of sweetened milk and vitamins was used as a reinforcer. All experimental events were programmed and recorded automatically by relay and timing circuits, except that on the final test day responses were recorded manually. Two Hewlett-Packard audio oscillators (Model 200AB) were used to generate tones of $45 \mathrm{~dB}$ re .002 dynes $/ \mathrm{cm}^{2}$ inside the chamber.

\section{BEHAVIORAL PROCEDURE}

Animals were shaped to respond differentially on a two-lever auditory discrimination task. After a 4-sec period, during which barpressing on either lever reset a timer (DRL $4 \mathrm{sec}$ ), either a high or a low tone was presented. A response on the right lever in the presence of a $1,000 \mathrm{~Hz}$ tone (high) and a response on the left lever in the presence of a $500-\mathrm{Hz}$ tone (low) were arbitrarily chosen as "correct." These were initially followed by milk on every "correct" trial (a noncorrection procedure was used), but gradually the ratio of unreinforced to reinforced trials was raised to 10:1 (FR 10). independently tabulated for each lever. Incorrect responses were followed by a 2-sec time-out (TO). during which the box was dark. Two hundred and forty discrete trials were given each day until a stable baseline (percent correct) was obtained. Generalization testing began under extinction conditions. Twelve different frequencies, including the training stimuli, $(200,300,400,500,600,700$, $800,900,1,000,1,100,1,200$, and $1.300 \mathrm{~Hz}$ ) were presented in random order such that 20 presentations of each frequency occurred, for a total of 240 discrete trials. Stimulus-on periods lasted until the animal responded on one of the two levers. Reaction time and the lever to which the animal responded were recorded for each trial.

After Day 1 of generalization testing, the animals were returned to the original baseline conditions. A second generalization test was given 7 days later, and a third generalization test followed another 7-day return to baseline.

PHARMACOLOGICAL PROCEDURE

On Day 1 of generalization testing, either $.16 \mathrm{mg} / \mathrm{kg} \mathrm{LSD}^{1}$ or $.05 \mathrm{cc}$ saline was administered intraperitoneally (IP) to each animal $1 / 2 \mathrm{~h}$ before testing. At the time of the second generalization test, animals previously receiving saline received $.16 \mathrm{mg} / \mathrm{kg} \mathrm{LSD}$, and animals previously receiving $\mathrm{LSD}$ received $.05 \mathrm{cc}$ saline. All three animals received $.04 \mathrm{mg} / \mathrm{kg}$ LSD immediately before the third generalization session.

\section{RESULTS}

Figure 1 shows the generalization gradients obtained for the three animals under control (saline) conditions. The relative probability of right-lever responses over the entire session is plotted as a function of stimulus frequency. (The probability of responses on the left lever is the complement of the probability of right-lever responses). It can be seen that each of the three animals produced regular gradients of similar form. The probability of a right response is greatest at $T_{2}$, the stimulus in the presence of which a right response was reinforced during training. This probability generally decreases as the distance from $T_{2}$ increases, except that the gradient flattens considerably at approximately $600 \mathrm{~Hz}$. When sessions were broken down into blocks of 80 trials, no consistent within-session differences were found.

Table 1

Average Percentages of Correct Responses During the Last 5 Days of Discrimination Training

\begin{tabular}{lcc}
\hline Animal & $1,000-\mathrm{Hz}$ Tone & $500-\mathrm{Hz}$ Tone \\
\hline 706 & 88 & 82 \\
707 & 92 & 85 \\
708 & 78 & 72 \\
\hline
\end{tabular}




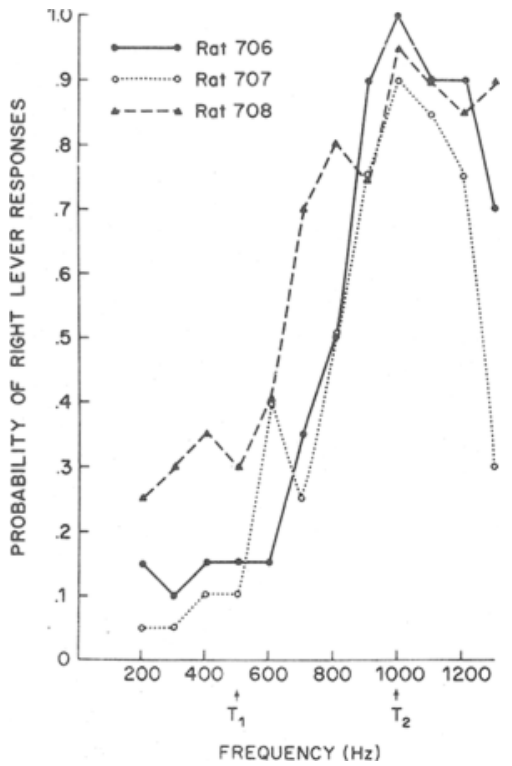

Fig. 1. Probability of right-lever responses as a function of each testing stimulus for three rats.

The data of Rat 708 deserve special mention, since its gradient is flatter and more irregular than those of the other animals. The difficulty of this case can probably be traced to this animal's lower level of accuracy during discrimination training (Table 1).

The effects of .04 and $.16 \mathrm{mg} \mathrm{LSD} / \mathrm{kg}$ on the generalization gradient of Animal 706 are shown in Fig. 2. Neither dose altered the shape of the gradient in a systematic or significant manner. Similar results were obtained in the other two-rats.

Figure 3 shows frequency distributions of reaction times under control and drug conditions. Reaction time was defined as the time from the onset of a tone until the animal responded. These times were calculated for all stimulus frequencies and divided into groups: very short $(<.5 \mathrm{sec})$, short $(.5-1.0 \mathrm{sec})$, intermediate $(1.0-10 \mathrm{sec})$, and long ( $>10 \mathrm{sec})$. Both .04 and $.16 \mathrm{mg} \mathrm{LSD} / \mathrm{kg}$ increased the percentage of long reaction times. The percentage of very short reaction times was slightly facilitated by $.04 \mathrm{mg} \mathrm{LSD} / \mathrm{kg}$ and decreased after $.16 \mathrm{mg} \mathrm{LSD} / \mathrm{kg}$. The percentage of short reaction times decreased after both .04 and $.16 \mathrm{mg}$ $\mathrm{LSD} / \mathrm{kg}$. Little variation is evident in the intermediate reaction times (not shown). DISCUSSION

The generalization gradients reported here resemble those of other investigators. Following differential training, there is usually a systematic shift in response probability as a function of the stimulus value between the two training points. For example, in one-response situations the rate of occurrence of the response decreases as

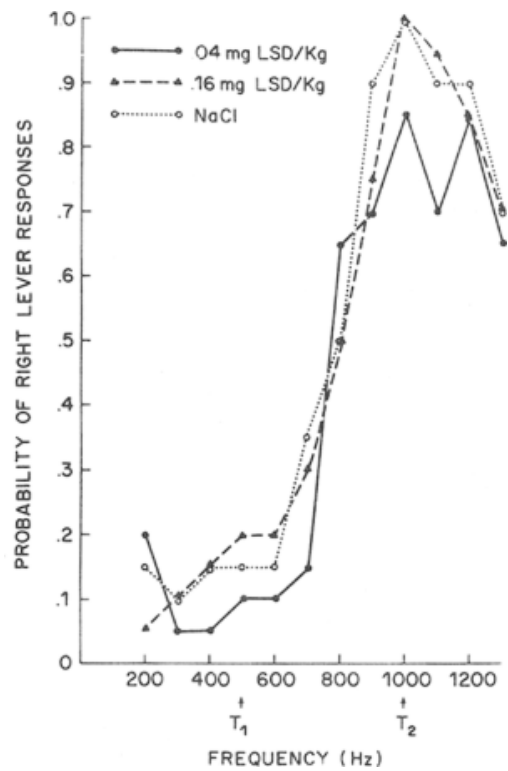

Fig. 2. Probability of right-lever responses as a function of each testing stimulus for Rat 706 under drug and control conditions.

the distance between the testing and training stimuli increases (e.g., Guttman \& Kalish, 1956; Pierrel, 1958); in other two-response situations, the probability of occurrence of one of the two responses gradually decreases as the probability of the other response increases (e.g., Honig \& Day, 1962; Migler \& Millenson, 1969; Risley, 1964).

At the extremes of the stimulus dimension (beyond $T_{1}$ and $T_{2}$ ), the gradients we obtained differ somewhat from those of previous studies involving two discrete responses. The usual result is that responding is maximum or minimum (depending on which response is being plotted) at the training stimuli and remains maximum (or minimum) at the extremes of the stimulus continuum (Cross \& Lane, 1962; Honig \& Day, 1962; Risley, 1964). In the data presented here, however, minimum responding does not occur at $T_{1}$. Rather, it has shifted to the stimulus values below the testing stimulus and flattens thereafter.

While this shift in response probability might indicate some sort of peak shift (Hanson, 1959), it is more likely that there is poor stimulus control in the presence of tones of low frequencies. It should be noted that all three animals were more accurate at the higher tone during discrimination training than at the lower tone (see Table 1). Furthermore, the rat audiogram suggests that at $45-\mathrm{dB}$ tones less than $500 \mathrm{~Hz}$. may be below the rat's auditory threshold (Gourevitch \& Hack, 1968).

The data around $T_{2}$ are somewhat different from those around $T_{1}$. Maximum

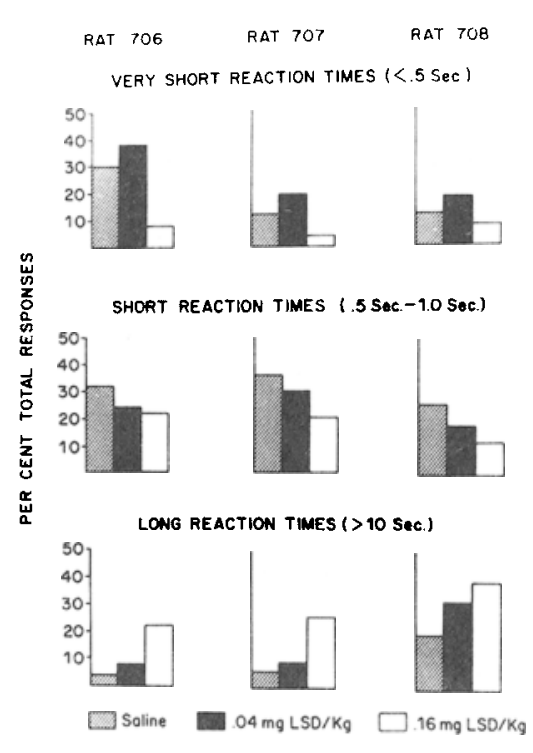

Fig. 3, Frequency distributions of reaction times for each stimuli (see text).

responding occurs at the training stimulus, but instead of flattening at the points beyond $T_{2}$, responding decreases as the distance from $T_{2}$ increases. This "tent-shaped" distribution of response probability resembles that obtained in studies which measure generalization in terms of rate of responding (e.g., Guttman \& Kalish, 1956).

The drug data indicate that even though LSD (at doses of .04 and $.16 \mathrm{mg} / \mathrm{kg}$ ) has a marked effect on the animal's reaction time to the presentation of a discriminatory stimulus, it does not alter stimulus control, as measured by the amount of generalization to other values of the discriminatory stimuli. Thus, these data are consistent with Key's (1961) results; in both avoidance and appetitive situations, LSD has no effect on the shape of the generalization gradient.

The reaction-time data following administration of LSD are also in agreement with those of previous investigators. The increase in long reaction times with both .04 and $.16 \mathrm{mg} \mathrm{LSD} / \mathrm{kg}$ ( $>10 \mathrm{sec}$ ) is consistent with reports that LSD produces periods of pausing or no responding (Appel, 1968; Becker et al, 1967; Berryman et al, 1962; Blough, 1956; Freedman et al, 1964; Jarvik \& Chorover, 1960; Peterson, 1966).

The facilitatory effect of $.04 \mathrm{mg} \mathrm{LSD} / \mathrm{kg}$ shown here by an increase in the percentage of very short reaction times ( $>.5 \mathrm{sec}$ ) has been reported with other measures (Jarrard, 1963); it suggests that LSD may produce an increased arousal or readiness to respond (Bradley \& Elkes, 1957; Bradley \& Key, 1959; Key \& 
Bradley, 1960).

That there is no consistent change between drug and control conditions in the distribution of intermediate reaction times seems to indicate that LSD does not change the normal rate of responding. Instead, it affects the overall rate indirectly by producing periods of no responding, which are averaged with periods of responding at the normal rate (Blough, 1963; Migler \& Millenson, 1969). Failure to respond in the presence of a given stimulus, however, can be caused by factors other than the loss of stimulus control (Blough, 1966). Therefore, a discrete trial procedure which allows the animal to make only one of two possible responses to each stimulus may be a more reliable way to assess stimulus control than to measure rate of responding.

\section{REFERENCES}

APPEL, J. B. The effects of "psychotomimetic" drugs on animal behavior. In D. Efron (Ed.), Psychopharmacolog: $A$ review of progress $1957-1967$. Washington, D.C: U.S. Government Printing Office, 1968. Pp. 1211-1222.

BECKER, D. I., APPEL, J. B., \& FREEDHAN, D. X. Some effects of LSD on visual disctimination in pigeons. Psychopharmacologia, 1967, 11, 354-364.

BERRYMAN, R., JARVIK, M. E., \& NEVIN, J. A. Effects of pentobarbital, lysergic acid diethylamide, and chlorpromazine on matching behavjor in the pigeon. Psychopharmacologia, 1962, 3, 60-65.

BLOUGH, D. S. Some effects of drugs on visual discrimination in the pigeon. Annais of the New York Academy of Sciences, 1956, 66, 733-739.

BLOUGH, D. S. Interresponse time as a function of continuous variables: $A$ new method and some data. Journal of the Experimental Analysis of Behavior, 1963, 6, 237-246.

BLOUGH, D. S. Definition and measurement in generalization research. In D. I. Mostofsky (Ed.), Stimulus generalization. Stanford: Stanford University Press, 1965. Pp. 30-37.

BLOUGH, D. S. The study of animal sensory processes by operant methods. In W. K. Honig (Ed.), Operant behavior: Areas of research and application. $\mathrm{Ne}$ W Y r k: Appleton-Century-Crofts, 1966. Pp. 345-379.

BRADLEY, P. B., \& ELKES, J. The effects of some drugs on the electric activity of the brain. Brain, 1957, 80, 77-117.

BRADLEY, P. B., \& KEY, B. J. A comparative study of the effects of drugs on the arousal system of the brain. British Joumal of Pharmacology, 1959, 14, 340-349.

CROSS, D. V., \& LANE, H. L. On the discriminative control of concurrent responses: The relations among response frequency, latency, and topography in auditory generalization. Joumal of the Experimental Analysis of Behavior, 1962, 5, 487-496.

FREEDMAN, D. X., APPEL, J. B., HARTMAN, F. R., \& MOLLIVER, M. E. Tolerance to behavioral effects of LSD-25 in rat. Journal of Pharmacology \& Experimental Therapeutics, $1964,143,309-313$.

GOUREVITCH, G., \& HACK, M. H. Audibility in the rat. Journal of Comparative \& Physiological Psychology, 1966, 62, 289-291.

GUTTMAN, N., \& KALISH, H. I.
Discriminability and stimulus generalization. Joumal of Experimental Psychology, 1956, 51, 79-88.

HANSON, H. M. Effects of discrimination training on stimulus generalization. Joumal of Experimental Psychology, 1959, 58, 321-334.

HOLLISTER, L. E. Human pharmacology of lysergic acid diethylamide. In D. Efron (Ed.), Psychopharmacology: A review of progress 1957.1967. Washington, D.C: U.S. Government Printing Office, 1968. Pp. 1253-1261.

HONIG, W. K., \& DAY, R. W. Discrimination and generalization of a dimension of stimulus difference. Science, 1962, 138, 29.31.

JARRARD, L. E. Effects of d-lysergic acid diethylamide on operant behavior in the rat. Psychopharmacologia, 1963, 5, 39.46.

JARVIK, M. E., \& CHOROVER, S. Impairment by lysergic acid diethylamide of accuracy in performance of a delayed alternation task in monkeys. Psychopharmacologia, 1960,1 , 229-230.

KEY, B. J. The effects of drugs on discrimination and sensory generalization of auditory stimuli in cats. Psychopharmacologia, 1961, 2, 352-363.

KEY, B. J., \& BRADLEY, P. B. The effects of drugs on conditioning and habituation to arousal stimuli in animals.
Psychopharmacologia, 1959-1960. 1, 450-462. MIGLER, B. Etfects of averaging datis during stimulus generalization. Journal of the Experimental Analysis of Behavior. 1964, 7. 303-307.

MIGLER, B., \& MILLENSON, J. R. Analysis of response rates during stimulus generalization. Journal of the Experimental Analysis of Bchavior, 1969, 12, 81-87.

PETERSON. N. J. Some effects of LSD on the control of responding in the cebus monkey by reinforcing and discriminative stimuli. Unpublished MD thesis, Yale Lniversity, 1966.

PIERREL, R. A generalization gradient for auditory intensity in the rat. Journal of the Experimental Analysis of Behavior, 1958, 1. 303-315.

RISLEY, T. Generalization gradients following two-response discrimination training. Joumal of the Experimental Analysis of Behavior, 1964, 1, 199.204.

TERRACE, H. S. Stimulus control. In W. K. Honig (Ed.), Operant beharior: Areas of research and application. New York: Appleton-Century-Crofts, 1966. Pp. 271-344. NOTE

1. Ampules of $0.1 \mathrm{mg} / \mathrm{ml}$ manufactured by Sandoz Pharmaceuticals were obtained from the National Institute of Mental Health, Center for Studies of Narcotics and Drug Abuse.

\title{
Extinction as a function of varied quality and quantity of reward sequence in a straight alley
}

\author{
RAYMOND RUSSIN, WILLIAM N. BOYER, and HENRY A. CROSS \\ Colorado State University, Fort Collins, Colo. 80521
}

Four groups of male albino rats were extinguished in a straight alley after receiving two daily trials on one of the following varied quality and/or quantity of reward sequences: 1 alfalfa pellet followed by 1 sucrose pellet, 1 sucrose pellet followed by 1 alfalfa pellet, a single alfalfa pellet followed by 16 sucrose pellets, and a single sucrose pellet followed by 16 sucrose pellets. The results indicated that a high-quality reward following a low-quality reward produced greater resistance to extinction than when a low-quality reward followed a high-quality reward. The findings were interpreted within Capaldi's sequential theory.

Capaldi (1967) has presented a hypothesis that large rewards following small rewards produce greater resistance to extinction than do large rewards followed by small rewards. According to Capaldi, the first reward provides a trace or "aftereffect," and the second reward conditions this trace in direct proportion with the magnitude of the second reward. Maximum resistance to extinction can therefore be achieved by employing a second reward greater in magnitude than the first reward. Leonard (1969) supported this prediction by showing that smaller rewards on the first trial of a two-trial reward sequence increased resistance to extinction.

Some investigators (e.g., Guttman, 1953; Hutt, 1954) have observed that rats prefer sweeter rewards to less sweet rewards.
Cross, Rankin, \& Wilson (1964) also found that rats receiving a dextrose reward made fewer errors in a multiple-Y maze than did rats receiving an alfalfa-base reward. The Guttman and Hutt studies reported that during extinction more responses were emitted when a preferred quality of reward was used during training.

These studies suggest that quantity and quality have some parallel effects on performance and even on learning measures. If the assumption is made that larger quantities of reward are more preferable than smaller quantities, and if sweeter rewards are more preferable than less sweet rewards, than an extension of Capaldi's sequential hypothesis can be made to include quality of reward. It is conceivable that more preferred rewards, either in terms of quantity or quality, 